\title{
PRINCIPLE MANAGEMENT OF SNAKE BITES WITH REFERENCE TO EGYPT \\ BY
}

TOSSON A. MORSY ${ }^{1 *}$ MAI KHATER A. KHATER ${ }^{2}$ AND

AHMED KHALIFA E. KHALIFA ${ }^{1}$

Faculty of Medicine, Ain Shams University ${ }^{1}$, Cairo 11566 and Military Medical Academy ${ }^{2}$, Cairo, 11291, Egypt ( ${ }^{*}$ Correspondence; morsyegypt2014@gmail.com)

Abstract

Snakebite is a serious and important medical emergency encountered in worldwide. Snakes are elongated, limbless, carnivorous reptiles of the suborder Serpentes. Like all other squamates, snakes are ectothermic, amniotic vertebrates covered in overlapping scales. Most species of snake are nonvenomous and those that have venom use it primarily to kill and subdue prey rather than for self-defense. Some possess venom enough to cause painful injury or death to humans. Nonvenomous snakes either swallow prey alive or kill by constriction. The epidemiology of snake envenomation varies widely between region and local knowledge is vital in determining treatment.

Key words: Snake, Bite management, Military Nursing role, Review.

\section{Introduction}

Snake bites account for 125,000 deaths annually worldwide (Chippaux, 1998). Venom from four snakes' families (Atractaspididae, Colubridae, Elapidae, \& Viperidae) is toxic to man (Thornton, 2014). Parrish (1966) in USA reported that about 8,000 venomous snakebites occurred annually. Juckett and Hancox (2002) in USA reported that venomous snakebites, although uncommon, were a potentially deadly emergency and rattlesnakes caused most snakebites fatalities. They added that venomous snakes were classified as having hemotoxic or neurotoxic venom. Al-Sadoon (2015) in Saudi Arabia reported that snakebites occurred throughout the six years with the highest frequency in 2005 and least in 2006 where most of the bite cases were mild and all cured except four patients who died after administration of antivenom during $24 \mathrm{hr}$ post snakebite. The majority was males $(81.7 \%)$ and the most attacked age was within $11-30$ years $(51.5 \%)$.

WHO (2019) reported that every year, about 5.4 million snakebites occurred worldwide, which caused up to 2.7 million envenomings, almost 138,000 deaths, and 400,000 cases of sequelae or disability, which was a neglected, occupational disease in subtropic al \& tropical countries in Asia, Africa, and Latin America. Bolon et al. (2020) reported that snakebite victims and healthcare provid- ers played a central role in biting snake identification and novel approaches (e.g. photographing snake, crowdsourcing) could help increase biting snake taxonomy collection to better understand their ecology and epidemiology and ultimately improve snake bite management.

Snake venom varies in potency and action (Barrington et al, 1984), but several syndromes were distinguished post envenomation: 1- Neurotoxicity, 2- Systemic toxicity as hypotension \& shock, 3- Coagulopathy, 4- Rhabdomyolysis, 5- Renal failure, and 6- Local tissue necrosis. The major aspects of snake bite treatment include field management, hospital assessment, and hospital management.

Field management: Initial first aid of envenomation was directed at reducing the spread of venom and expediting transfer to an appropriate medical center.

General principles: The general principles were agreed upon (Gold et al, 2002): 1-The patient should be removed from the snake's territory, kept warm and at rest, and be reassured. 2- Injured body part should be immobilized in a functional position below the heart level. 3- Wound should be cleansed, except in areas where a venom detection kit was used in Australia. 4- Withhold alcohol $\&$ drugs that may confound clinical assessment. 5- Attempt to identify the snake, without endangering the patient or rescuer. A digital 
photo taken at a safe distance may be useful. Snake parts should not be handled directly. The bite reflex may remain intact in recently killed snakes, possibly being able to inflict a bite, and 6- Transport the patient to the nearest medical facility as quickly as possible.

Pressure immobilization: The pressure immobilization technique to delay the systemic absorption of snake venom has widespread use in Australia, where elapid toxin primarily causes neurotoxicity without tissue necrosis and where there may be significant delaysed in transfer to medical facilities (Cheng and Currie, 2004). Its efficacy was first detected in studies on monkeys using tiger snake venom (Notechis scutatus) with high systemic toxicity (Sutherland et al, 1979). Immobilization of the envenomed limb and application of pressure of $55 \mathrm{mmHg}$ resulted in a significant delay in systemic venom absorption. Dramatic benefits were given in the anecdotal reports (Pearn et al, 1981). But, pressure immobilization value against venoms was unproven. Some venoms; such as cobra and viper venom, primarily produce local necrosis; in this setting, localization of toxin may worsen the syndrome (Murrell, 1981). Other potential problems with this technique include the availability of suitable materials and difficulty to determine the appropriate pressure to apply. If the compression bandage was too loose, it would not work, or if too tight, it might obstruct arterial flow. Another concern was that release of compression bandage may cause sudden systemic envenomation. Thus, compression bandages, if used, should not be removed until the patient reached the hospital and a clinical assessment of the patient has occurred.

Generally, pressure immobilization techniquee was recommended following envenomation by snakes with neurotoxic venom, including Australian elapids. It was usually not advised following snakebites with locally necrotic venom, as the cobras and vipers.

Not recommended methods: Methods such as incision and oral suction, mechanical suction devices, cryotherapy, surgery, and electric shock therapy have been widely used, but are no longer recommended. As an example, a common misconception is that one should apply a tourniquet, suck out the poison, and spit it out. But, this was strongly discouraged, since it damaged nerves, tendons, and blood vessels and led to infection (Hall, 2001). Moreover, venom removal by suction was minimal. This was given in a study of mock venom extraction with a mechanical suction device in human volunteers; suction reduced total body venom burden by only $2 \%$ (Alberts et al, 2004).

Hospital assessment: Variation in toxin venom components occurred both inter- and intra- specifically resulted of various processes, including gene duplication and action of positive selection (Casewell et al, 2020). The evaluation of the patient should take into account each syndrome (Tab. 1).

Bite evaluation: There is considerable variation in the locally necrotic action of snake venom. In Australia, since little local necrosis and minimal local pain were common; fang marks may easily be missed. In contrast, cobras and vipers cause extensive local tissue destruction.

The first clinical indications of systemic envenomation are often nonspecific symptoms such as nausea, vomiting, abdominal pain, and headache. The presence of the symptoms should prompt an intensive assessment for the other clinical syndromes associated with snake envenomation.

Neurotoxicity: Venoms of the elapids of the Australia-Pacific region are associated with severe neurotoxicity. Neurotoxicity is characterized initially by ptosis, diplopia, \& bulbar palsy with onset between 1 to $10 \mathrm{hrs}$ following envenomation. Symptoms can later progress to dysarthria and more generalized weakness. Thus, frequent, serial observations for ptosis, diplopia, and impaired swallowing function were of paramount importance. Ventilatory support was required for airway protection or respiratory paralysis.

Coagulopathy: Venom may be either a procoagulant or an anticoagulant, but both ga- 
ve similar coagulation disorders rapidly. Overt bleeding signs should be sought following a snake bite. Low-grade bleeding from gums and epistaxis are common (Mosquera et al, 2003). Stroke resulting primarily from intracranial hemorrhage complicated Bothrops spp. snake bites in $2.6 \%$ of cases in a series from Ecuador and was a well-recognized complication following Australian elapid and certain other bites (Otero et al, 2002).

The whole blood clotting test (WBCT) is a useful bedside screening test; failure of the blood to clot in a clean glass tube after 20 minutes is evidence of severe hypofibrinogenemia. Where available, coagulation parameters as INR, aPTT, fibrinogen, and fibrin degradation products must be taken at baseline (Sano-Martins et al, 1994).

The optimal management of asymptomatic patients with laboratory evidence of coagulation defects was uncertain. Extrapolation from other settings suggested that a multicomponent coagulopathy with parameters exceeding critical thresholds (INR >3.0, aPTT $>50$ seconds, platelets $<50,000 /$ microL, and fibrinogen $<75 \mathrm{mg} / \mathrm{dL}$ ) was associated with a major bleeding risk of $1 \%$ over a few days (Yip, 2002).

Clinical assessment, the WBCT and other clotting parameters should be followed serially. The presence of coagulopathy may be useful in the clinical diagnosis of snake bite. In Papua New Guinea, for example, envenomation from the death adder rarely causes a significant coagulopathy in contrast to that of the taipan.

Hypotension and shock: Hypotension and shock may result from a snake bite (Udayabhaskaran et al, 2017). The mechanisms for these findings include: vasodilation, a direct action of venom on myocardium, and/or hypovolemia due to bleeding. Following a snake bite, assessment of fluid status clinically, or where available, with central monitoring is important.

Rhabdomyolysis: Muscle pain, weakness, and dark urine were suggestive that massive rhabdomyolysis has occurred. Creatine kina- se and electrolytes, particularly hyperkalemia, should be followed serially, and the myoglobinuria presence must be documented. Urine dipstick tests for presence of blood detected in myoglobin and hemoglobin (as well as hematuria) and positive results were suggestive of significant rhabdomyolysis.

Renal failure: Renal failure after a snake bite was multifactorial resulted from hypotension, rhabdomyolysis and/or disseminated intravascular coagulation (Iba and Levy, 2020). In 100 Crotalus durissus envenomed patients, 29 developed acute renal failure (ARF) due to rhabdomyolysis. Independent risk factors for ARF included age $<12$ years (odds ratio [OR] 5.6, 95\% CI 1.2-26.2), a delay in antivenom therapy of $>2 \mathrm{hrs}$ (OR $11.1,95 \%$ CI 1.2-101), \& a creatinine kinase at admission $>2000 \mathrm{U} / \mathrm{L}$ (OR 12.7, 95\% CI 2.8-58), in contrast to diuresis at admission $>90 \mathrm{ml} / \mathrm{hr}$. (OR $0.2,95 \%$ CI $0.05-0.7$ ) was protective (Pinho et al, 2005).

Hospital management: Snakebites are rare medical emergency cases in Europe but may sometimes be severe and led to complications, and children and male victims were much affected, contrary to what one would expect given their respective proportion in the entire population. Both upper and lower limb bites were recorded at an equal frequency while the bites in other body parts were very rare (Chippaux, 2012). But, time required for hospital observation before discharge of an asymptomatic patient with possible envenomation was a local issue (Currie, 2003). Each hospital where admissions for snake bites occur should assess and prospectively evaluate its own discharge criteria based upon the venom properties of the local snake fauna and the level of clinical and laboratory expertise available.

Oxygen should not routinely be administered to patients with suspected neurotoxic envenomation. Although other clinical features of neurotoxicity usually manifest earlier (particularly bulbar palsy), hypoxia due to hypoventilation mandates intubation. Detection of hypoxia would be impaired by oxyg- 
en therapy. The tetanus toxoid need must be assessed. (Tetanus is a nervous system disorder characterized by muscle spasms can be used by toxin-producing anaerobe, Clostridium tetani found in the soil (CDC, 2020). The clinical features of tetanus and its relationship to traumatic injuries were known to the ancient Egyptians and Greeks and too many clinicians before the introduction of vaccination with tetanus toxoid in 1940s. In late 1940 s, tetanus toxoid-containing vaccines were introduced into routine childhood vaccination and tetanus became a well known disease. At that time, about 500-600 cases (about 0.4 cases $/ 100,000$ ) were annually reported (Roper et al, 2018). Tetanus-diphtheria toxoid vaccination adults was higher than diphtheria-specific antibodies, and a substantial proportion of them, and particularly of elderly ones, did not have protective antibody concentrations against diphtheria (Weinberger, 2017)

In Brazil bites by many species of venomous snake resulted in local necrosis at, or extending from the bite site, but prophylactic antibiotics to prevent infection as a complication of local necrotic envenoming was controversial even with chloramphenicol (Jorge et al, 2004). But, a double-blind randomized controlled trial in 251 patients following $\mathrm{Bo}$ throps envenomation in Brazil, found that oral chloramphenicol (500mg every six hrs for five days) did not prevent abscess formation as $4.9 \%$ developed abscess in the chloramphenicol group compared with $4.7 \%$ in the placebo group (Jorge et al, 2004) Also, in Sri Lanka among 144 patients no significant difference was in swelling in patients randomly assigned to benzylpenicillin and metronidazole compared with placebo (Kularatne et al, 2005). Where possible, the snake should be identified clinically, by examination or by venom kit testing. Local knowledge was vital; in Southeast Asia, for example, syndromic diagnosis was used (Coppola and Hogan, 1992). In Australia, snakes were very widely in appearance, and identification was rarely possible by the clinician (Mo- rrison et al, 1983); however, venom detection kits were useful in determining the appropriate monovalent antivenom (Sutherland, 1992). If there was doubt about the snake identity, treatment was administered for an unidentified snake bite (Jelinek et al, 1991).

Antivenom: The use of antivenom depends upon availability and the risk-benefit ratio; thus, this was a clinical decision that must be made for each patient on an individual basis. Any decision to use antivenom must be taken into account association of most antivenom with a high incidence of allergic reactions. Antivenoms are generally indicated when (Warrell, 1990): a- There was evidence of systemic envenomation (neurotoxicity, coagulopathy, rhabdomyolysis, persistent hypotension, and/or renal failure), or bThere was severe local envenomation, manifested by local tissue destruction. Although delayed in administration gave lowered effectiveness, anecdotal evidence suggested that some improvement was possible even days after envenomation with some snakes.

Preparation, dosing, and administration of antivenom should follow established local product information guidelines; dosing was generally the same for children and adults.

Adverse reactions: Reactions to most antivenom are common and may be divided into three types: a- Early allergic reactions, bPyrogenic reactions, $7 \mathrm{c}$ - Late allergic reactions (serum sickness). The rate of early and late allergic reactions varies with different antivenom preparations and depends on the method of purification, total foreign protein load and the composition of antivenom of whole immunoglobulin compared 7o Fab fragments (Lalloo and Theakston, 2003). Drug hypersensitivity reactions (DHR) constitute a major and common public health problem, particularly in children. One of the most severe manifestations of DHR is anaphylaxis, which might be associated with a lifethreatening risk (Lieberman, 2012). Prompt assessment and treatment were critical in anaphylaxis, as respiratory or cardiac arrest \& death can occur within minutes (Pumphrey, 
2000). The cornerstones of initial management were the following (Brown, 2005): 1-Re moval of inciting antigen, if possible (e.g., stop infusion of a suspect medication), 2Call for help (summon a resuscitation team in the hospital setting, call 911 or an equivalent service in community setting), 3- Intramuscular injection of epinephrine, 4- Patient placement in the supine position with the lower extremities elevated, or if dyspnea or vomiting, placement of patient semi-recumbent with lower extremities elevated, 5- Oxygen supplemental, \& 6- Volume resuscitation with intravenous fluids

The use of prophylactic epinephrine prior to the administration of antivenoms showed high rates of controversial allergic reactions. A randomized, placebo-controlled trial of 105 Sri Lankan patients demonstrated a significant reduction in adverse events due to antivenom, without reported adverse events ascribed to the use of epinephrine as $0.25 \mathrm{ml}$ of $0.1 \%$ solution subcutaneously (Premawardhena et al, 1999). Also, among 52 patients from the same center, the frequency of mildto-moderate early reactions was reduced by the combination of an antihistamine bolus and a corticosteroid infusion (Gawarammana et al, 2004). But, rate of acute reactions to polyvalent antivenom in the control arm in both studies was extremely high $(>40 \%)$. As a result, these benefits should be interpreted with caution in other regions.

N.B.: Route of epinephrine administration depends upon symptoms. For patients who are not profoundly hypotensive or in shock or cardiorespiratory arrest, IM injection into the mid-anterolateral thigh as an initial route of administration was recommended, in preference to subcutaneous one. For adults, the dose of epinephrine is $0.3 \mathrm{mg}$ to $0.5 \mathrm{mg}$, IM injected into the mid-anterolateral thigh that might be repeated at 5 to 15 minute interva1s. For infants \& children, epinephrine dose was IM $0.01 \mathrm{mg} / \mathrm{kilogram}$ up to $0.5 \mathrm{mg} / \mathrm{dose}$, injected into the mid-anterolateral thigh. Treatment may be repeated at 5 to 15 minute intervals. IV epinephrine was indicated for patients with profound hypotension or symptoms \& signs suggestive of impending shock (dizziness, incontinence of urine or stool) or those who do not respond to an initial IM injection of epinephrine and fluid resuscitation. For these patients it was suggested that epinephrine be given by the continuous slow intravenous infusion rather than by intermittent IV bolus. Massive fluid shifts can occur in anaphylaxis, and patients with orthostasis, hypotension, or incomplete response to epinephrine must receive large volume fluid resuscitation with normal saline. Normotensive patients must receive normal saline to maintain venous access in case their status deteriorates. It was critical that patients be more evaluated to confirm the trigger, as specific avoidance measures were useful in reducing the recurrence risk. Also, for some allergens, immunomodulation was available to reduce risk.

Prophylactic antihistamines alone were not beneficial in the Brazilian study (Fan et al, 1999), but, corticosteroids were used with early and late allergic reactions. Although no definite data, when premedication with epinephrine was accepted in the following cases (Cheng and Winkel, 2004): a- In areas where the use of antivenom was associated with high rates of allergic reactions, and b- In areas where there was an appreciable risk of allergic reaction associated with antivenom use and the management of acute allergic reactions was problematic because of limited staffing or facilities.

If premedication was lacking, epinephrine ( 0.5 to $1 \mathrm{ml}$ of $0.1 \%$ or $1: 1000$ solution) must be prepared prior to administration of any antivenom in the event that it was needed.

Failure to respond: Failure of response to antivenom may be due to the wrong monovalent antivenom, insufficient antivenom, inactive antivenom or an excessive delay in administration after envenomation. Expired antivenom, sometimes used by veterinarians, retained its potency for some time following its use-by date, but risk-benefit ratio still supported its use if no "in date" antivenom was 
available (Christensen, 1975). WHO (2018) reported that the most effective envenomation treatment was the antivenom serum.

Specific complications management: Management of complications was by the adequate amounts of antivenom given. The following points were useful when considering the specific syndromes.

Local: Compartment syndromes may be confused with local necrosis appearance following envenomation but being rare. Fasciotomies should only be performed following confirmation of raised intracompartmental pressure with a Stryker needle (Stryker Instruments, Kalamazoo, MI) or IV cannula and pressure transducer and correction of coagulopathy (Wilson et al, 1997).

Neuropathy: Response degree in neuropathic symptoms depended upon the administration of antivenom time. Intubation and ventilation were required for airway protecti on or respiratory support if bulbar palsy, increasing dyspnea, or respiratory failure was present (Hudson, 1988).

Postsynaptic neurotoxins, which were present in venom of death adder (Acanthophis spp) and the Philippine cobra (Naja philippinensis), may be fully or partially reversed by an anticholinesterase such as edrophonium (10mg in adults) or neostigmine (50 to 100 $\mathrm{mcg}$ ), which was particularly useful in settings without antivenom available (Flachsenberger and Mirtschin, 1994).

Coagulopathy: It was not possible to correct coagulation defects without sufficient antivenom; and thus, persisting bleeding must prompt the administration of additional antivenom. Clotting factor replacement with the whole blood or fresh frozen plasma was only indicated in life-threatening cases hemorrhage after given antivenom. Although spontaneous bleeding resolved rapidly after antivenom, laboratory abnormalities might resolve more slowly. Neither heparin nor aminocaproic acid was effective (Tun, 1992).

Hypotension: Central pressure monitoring may be useful in the titration of intravenous fluid in patients who have not responded to fluid challenge. Central lines should be inserted with caution in patients with significant coagulopathy. The etiology of hypotension was multifactorial (Kelen et al, 1978).

Rhabdomyolysis: There was limited published evidence as to management of rhabdomyolysis following snake bite, and recommended approach was extrapolated from crush injuries (Smith and Greaves, 2003). Plasma volume expansion with intravenous isotonic saline should be given as soon as possIble. Treatment after initial fluid resuscitation often was alkaline-mannitol diuresis to urine $\mathrm{pH}$ of 6.5 . But, there was no clear clinical evidence that the alkaline diuresis was more effective than a saline diuresis; with a potential risk to alkalinization, since it promoted precipitation of calcium phosphate and induce or exacerbate hypocalcemia, and mannitol was ineffective if renal injury already occurred. At a minimum, optimization of fluid status and avoidance of drugs known to adversely affect renal function were of primary importance in the management of significant rhabdomyolysis.

Renal failure: The urine output should be monitored closely, since oliguria in patients who have been adequately volume replete usually portends significant renal failure (Al-Homrany, 1996). Central pressure monitoring may be useful in titrating fluid therapy. Short-term dialysis may be required.

The pathogenic mechanism of snake-bite induced acute kidney injury (AKI) remains unclear, and clinical manifestations of kidney damage consisted of acute kidney injury (AKI), hematuria, and proteinuria (Sitprija, 2008). The snake-bite induced AKI was associated with disseminated intravascular coagulation (DIC), intravascular hemolysis, \& rhabdomyolysis, of which DIC caused hemoglobin deposition in kidney tubules and led to degenerative necrosis (Mittal, 1994). Wei et al. (2016) in China reported that age, delayed antivenin therapy, creatine kinase, blood myoglobin, older age, regional lymphadenopathy, incision drainage, and hemoglobin were risk factors for snakebite induced AKI 
and early treatment induced AKI and impet al. (2021) in India found that apart from the fluid \& electrolyte management, kidney support as the early and prompt initiation of kidney replacement therapy when indicated forms the bedrock in management of snake bite associated AKI. Long-term follow-up was important due to chance of the CKD progression.

Cobra spit ophthalmic: Venom of spitting cobras (Naja spp.) found in Asia and Africa can cause corneal damage if introduced into the eye. Acutely, eye must be irrigated with copious quantities of water or saline. Slit la$\mathrm{mp}$ examination and fluorescein staining must be done to prove corneal ulceration; topical antibiotic ointment must be used if present. Diluted antivenom instillation was not recommended (Blackman and Dillon, 1992).

Other resources: Websites provide information about specific regions or snake species include: 1- A database and image library to assist clinicians with identified the most important venomous snakes by country or area and found antivenom products (file://apps. who.int/blood products/snake antivenoms/da tabase). 2- A global directory of poisons information centers (www.who.int/gho/phe/che mical_safety/poisonscentres/en/index.html).

In Egypt, since ancient times, Egypt had a complex relationship with its snakes. Most were harmless and even beneficial; preying on rodents and other pests, some, as cobra and the horned viper, were deadly, which venom consisted of neurotoxins and cytotoxins (Joubert and Taljaard, 1978). This lovehate relationship with snakes was reflected in ancient Egyptian mythology, with snakes represented both evil demons, such as the chaotic snake God Apophis, and benevolent gods such as the fertility Goddess Renenutet, who was often depicted in a cobra fo$\mathrm{rm}$. The commonest ones were: 1- The Horned Viper (highly venomous desert snake), 2-The Egyptian Cobra (another deadly one), 3- The African Egg-Eating Snake (more benevolent species), 4-The Kenyan Sand Boa (a good rodent hunter). roved prognosis of snakebite patients. Sarkar Now, what about the Military Nurses role? Snakebites can be lifelong consequences and is one of the main causes of death among military troops worldwide. Nursing care during and after antivenin treatment was continual patient assessment, monitoring for systemic complications, and follow-up treatment response (Dart, 2010). Patient's wound must be kept clean and covered with a sterile dressing. Debridement may be indicated if tissue necrosis was present. WHO (2018) reported that 4.5 to 5.4 million people experience snakebites annually, with 1.8 to 2.7 million of which become envenomated with mortality wranged between $81,000 \& 138,000$.

Avau et al. (2016) in Belgium reported the practical recommendation for snakebite treatment in a first aid setting was to immobilize the victim, while awaiting the emergency services. But, given the low to very low quality of data collected, high quality randomized controlled trials concerning the efficacy and feasibility of different variations of pressure immobilization technique were warranted. Parker-Cote and Meggs (2018) in USA suggested that pressure immobilization bandages and related strategies were the best interventions to delay systemic toxicity onset from venomous snakebites but may increase local toxicity for venoms that destroy tissue at the bite site, so must be individualized use to circumstances and venom nature.

CDC (2021) stated that workers should take these steps if a snake bites them: 1- Seek medical attention as soon as possible or call local Emergency Medical Services: a- Antivenom is the treatment for serious snake envenomation, and the sooner antivenom can be started; the sooner irreversible damage from venom can be stopped. b- Driving one to the hospital is not advised because people with snakebites can become dizzy or pass out. 2- Take a photograph of the snake from a safe distance if possible. Identifying the snake can help with treatment of snakebite. 3- Keep calm. 4- Inform your supervisor. 5Apply first aid on waiting for EMS staff to 
get you to the hospital: a-Lay or sit down with the bite in a neutral position of comfort, b- Remove rings and watches before swelling starts, c- Wash bite with soap and water, d- Cover bite with a clean, dry dressing, \& e- Mark the leading edge of tenderness/swelling on the skin and write the time alongside it. But, never do any of the following: 1- pick up the snake or try to trap it. Never handle a venomous snake, not even a dead one or its decapitated head, 2- wait for symptoms to appear if bitten, get medical help right away, 3- apply a tourniquet, 4- slash wound with a knife or cut it in any way, 5try to suck out venom, 6- apply ice or immerse wound in water, 7- drink alcohol as a painkiller, 8- take pain relievers (as aspirin), 9-Do not use electric shock or folk therapies.

\section{Conclusion}

1- Field management to the delay systemic absorption of venom and expediting transport to hospital. 2- Pressure immobilization only used for envenomation where risk of local necrosis was low and high consequences systemic toxicity (e.g., neurotoxicity).

Clinical data: Envenomation syndromes include local and systemic features. Major syndromes of systemic envenomation were neurotoxicity, coagulopathy, shock, rhabdomyolysis, and renal failure.

Evaluation and management: 1-Knowledge of the toxicity profiles of local snake species is vital, and expert advice should be sought if snake bite is uncommon. Clinical and laboratory assessment should be focused on specific syndromes, including local effects, paralysis, coagulopathy, shock, muscle damage, and renal failure. 2- Antivenom is indicated with significant local envenomation or evidence of systemic envenomation but may be accompanied by significant allergic phenomena. Decision to give antivenom must be based on risks and benefits of administration. 3-Complications of envenomation and administration of antivenom must be anticipated. Ongoing management was supportive.

\section{References}

Alberts, MB, Shalit, M, LoGalbo, F, 2004: Su- ction for venomous snakebite: A study of "mock venom" extraction in a human model. Ann. Emerg. Med. 43:181-4.

Al-Homrany M, 1996: Acute renal failure following snake bites: A case report and review. Saudi J. Kidney Dis. Transpl. 7:309-12.

Al-Sadoon, MK, 2015: Snake bite envenomation in Riyadh province of Saudi Arabia over the period (2005-2010). Saudi J. Biol. Sci. 22, 2:198-203.

Avau, B, Borra, V, Vandekerckhove, P, De Buck, E, 2016: The treatment of snake bites in a first aid setting: A systematic review. PLoS Negl. Trop. Dis. Oct 17;10(10):e0005079.

Barrington, PL, Condrea, E, Soons, KR, Yang, CC, Rosenberg, P, 1984: Effect of carboxylate group modification on enzymatic and cardiotoxic properties of snake venom phospholipases A2. Toxicon 22, 5:743-58.

Blackman, JR, Dillon, S, 1992: Venomous snakebite: Past, present, and future treatment options. J. Am. Board Fam. Pract. 5:399-404.

Bolon, I, Durso, AM, Mesa, SB, Ray, N, Alcoba, G, et al, 2020: Identifying the snake: First scoping review on practices of communities and healthcare providers confronted with snakebite across the world. PLoS One 15:e0229989.

Brown, SG, 2005: Cardiovascular aspects of anaphylaxis: implications for treatment and diag nosis. Curr. Opin. Allergy Clin. Immunol. 2, 5: 359-65.

Casewell, NR, Jackson, TNW, Laustsen, AH, Sunagar, K, 2020: Causes and consequences of snake venom variation: Review. Trends Pharmacol. Sci. (TIPS) 41, I8:570-81

CDC, 2020: Tetanus: Epidemiology and Prevention of Vaccine-Preventable Diseases.

Cheng, AC, Currie, BJ, 2004: Venomous snakebites worldwide with a focus on the AustraliaPacific region: Current management and contriversies. J. Intensive Care Med. 19:259-63.

Cheng, AC, Winkel, KD, 2004: Antivenom efficacy, safety and availability: Measuring smoke. Med. J. Aust. 180:5-8.

Chippaux, JP, 1998: Snake-bites: appraisal of the global situation. Bull WHO 76:515.

Chippaux, JP, 2012: Epidemiology of snakebites in Europe: a systematic review of the literature. Toxicon 59, 1:86-99.

Christensen, PA, 1975: The stability of refined antivenin. Toxicon 1, 13:75-8.

Coppola M, Hogan DE, 1992: Venomous snakes of southwest Asia. Am. J. Emerg. Med. 10, 3: 
230-6.

Currie, BJ, 2003: Snakebite in Australia: Moving from anecdotes to prospective studies. Emerg. Med. (Fremantle), 15:406-9.

Dart, RC, 2010: Goldfrank'sToxicologic Emergencies: Medical Toxicology. $9^{\text {th }}$ edition. Philadelphia, Lippincott, Williams \& Wilkins.

Fan, H, Marcopito, L, Cardoso, J, et al, 1999: Sequential randomised and double blind trial of promethazine prophylaxis against early anaphylactic reactions to antivenom for Bothrops snake bites. BMJ 318:1451-4.

Flachsenberger, W, Mirtschin, P, 1994: Anticholinesterases as antidotes to envenomation of rats by the death adder (Acanthophis antarcticus). Toxicon 32:35-9.

Gawarammana, IB, Kularatne, SA, Dissanayake, WP, et al, 2004: Parallel infusion of hydrocortisone $+/$ - chlorpheniramine bolus injection to prevent acute adverse reactions to antivenom for snake-bites. Med. J. Aust. 180:20-6.

Gold, BS, Dart, RC, Barish, R, 2002: Bites of venomous snakes. N. Engl. J. Med. 347:34-52.

Hall, EL, 2001: Role of surgical intervention in the management of crotaline snake envenomation. Ann. Emerg. Med. 37:175-9.

Hudson BJ, 1988: Positive response to edrophonium in death adder (Acanthophis antarcticus) envenomation. Aust. N Z J. Med. 18:792-7.

Iba, T, Levy, JH, 2020: Sepsis-induced coagulopathy and disseminated intravascular coagulation. Anesthesiology 132, 5:1238-45.

Jelinek, GA, Hamilton, T, Hirsch, RL, 1991: Admissions for suspected snake bite to the Perth adult teaching hospitals, 1979 to 1988. Med. J. Aust.155:761-6.

Jorge, MT, Malaque, C, Ribeiro, LA, et al, 2004: Failure of chloramphenicol prophylaxis to reduce the frequency of abscess formation as a complication of envenoming by Bothrops snakes in Brazil: a double-blind randomized controlled trial. Trans. R. Soc. Trop. Med. Hyg. 98:529-34.

Joubert, FJ, Taljaard, N, 1978: Naja haje haje (Egyptian cobra) venom: Some properties \& complete primary structure of 3 toxins (CM-2, CM -11 \& CM-12). Eur. J. Biochem. 90, 2:359-67.

Juckett, G, Hancox, JG, 2002: Venomous snakebites in the United States: Management review and update. Am. Fam. Physician 65, 7: 1367-75.

Kelen, EM, Rosenfeld, G, Vainzof, M, Machado, ZC, 1978: Experimental defibrination and bothropase: A study on the fibrinolytic mechanism in vivo. Haemostasis 7:35-9.
Kularatne, SA, Kumarasiri, PV, Pushpakumara, SK, et al, 2005: Routine antibiotic therapy in the management of the local inflammatory swelling in venomous snakebites: Results of a placebo-controlled study. Ceylon Med. J. 50: 151-6.

Lalloo, DG, Theakston, RD, 2003: Snake antivenoms. J. Toxicol. Clin. Toxicol. 41:277-80.

Lieberman, P, 2012: The risk and management of anaphylaxis in the setting of immunotherapy. Am. J. Rhinol. Allergy 26, 6:469-74

Mittal, BV, 1994: Acute renal failure following poisonous snake bite. J. Postgra. Med. 40:123-6. Morrison, JJ, Pearn, J, Covacevich, J, Nixon, J, 1983: Can Australians identify snakes? Med. J. Aust. 2:66-9.

Mosquera, A, Idrovo, LA, Tafur, A, Del Brutto, OH, 2003: Stroke following Bothrops spp. snakebite. Neurology 2003; 60:1577-82.

Murrell, G, 1981: The effectiveness of the pressure/immobilization first aid technique in the case of a tiger snake bite. Med. J. Aust. 2:295-8.

Otero, R, Gutiérrez, J, Beatriz Mesa, M, et al, 2002: Complications of Bothrops, Porthidium, and Bothriechis snakebites in Colombia: A clinical and epidemiological study of 39 cases attended in a university hospital. Toxicon 40:1107-9.

Parrish, HM, 1966: Incidence of treated snakebites in the USA. Pub. Hlth. Rep. 81:269-76.

Parker-Cote, J, Meggs, WJ, 2018: First aid \& prehospital management of venomous snake bites. Trop. Med. Infect. Dis. Apr 24;3 (2): 45. doi: 10.3390/tropicalmed3020045.

Pearn, J, Morrison, J, Charles, N, Muir, V, 1981: First-aid for snake-bite: Efficacy of a constrictive bandage with limb immobilization in the management of human envenomation. Med. J. Aust. 2:293-8.

Pinho, FM, Zanetta, DM, Burdmann, EA, 2005: Acute renal failure after Crotalus durissus snake-bite: A prospective survey on 100 patients. Kidney Int. 67:659-64.

Premawardhena, AP, de Silva, CE, Fonseka, MM, et al, 1999: Low dose subcutaneous adrenaline to prevent acute adverse reactions to antivenom serum in people bitten by snakes: Randomized, placebo controlled trial. BMJ 318:10414.

Pumphrey, RS, 2000: Lessons for management of anaphylaxis from a study of fatal reactions. Clin. Exp. Allergy 30:1144-9.

Roper, M, Wassilak, S, Scobie, H, 2018: Tetanus Toxoid. In: Plotkin, S, Orenstein, W, et al, 
(Eds.) Plotkin's Vaccines: $7^{\text {th }}$ edition, Elsevier. Sano-Martins, IS, Fan, HW, Castro, SC, et al, 1994: Reliability of the simple 20 minute whole blood clotting test (WBCT20) as an indicator of low plasma fibrinogen concentration in patients envenomed by Bothrops snakes. Butantan Institute Antivenom Study G. Toxicon 32:1045-8.

Sarkar, S, Sinha, R, Chaudhury, AR, Maduwage, K, Abeyagunawardena, A, et al, 2021: Snake bite associated with acute kidney injury. Pediatr. Nephrol. 2; Feb 9. doi: 10.1007/s00467020-04911-x.

Sitprija, V, 2008: Animal toxins and kidney: Nature clinical practice. Nephrology 4:616-27.

Smith, J, Greaves, I, 2003: Crush injury and crush syndrome: a review. J. Trauma 54:S226-3.

Sutherland, SK, 1992: Antivenom use in Australia: Premedication, adverse reactions and the use of venom detection kits. Med. J. Aust. 157: 734-8.

Sutherland, SK, Coulter, AR, Harris, RD, 1979: Rationalization of first-aid measures for elapid snakebite. Lancet 1:183-5.

Thornton, SL, 2014: Snakes in Encyclopedia of Toxicology ( $3^{\text {rd }}$ Edition).

Tun, PE, 1992: Heparin therapy. In: Russell's Viper Bite Victims with Disseminated Intravascular Coagulation: A controlled Trial. Southeast Asian J. Trop. Med. Publ. Hlth. 23:282.

Udayabhaskaran, V, Thomas, ETA, Shaji, B, 2017: Capillary leak syndrome following snakebite envenomation. Indian J. Crit. Care Med. 21, 10:698-702.

Warrell, D, 1990: Treatment of snake bites in the Asia Pacific region: A personal view. In: Snakes of Medical Importance (Asia Pacific Region), Gopalakrishnajone, P, Chao, L (Eds.), National University of Singapore, Singapore.

Warrell, DA, 2002: Bites of venomous snakes. N. Engl. J. Med. 347:1804-8.

Wei, Li, Chen, F, Shukun Wu, S, 2016: The related risk factors analysis of snakebite induced acute kidney injury. Med. Sci. Monit. Jul 5; 22:2335-9.doi: 10.12659/ msm.899072.

Weinberger, B, 2017: Adult vaccination against tetanus and diphtheria: The European perspective. Clin. Exp. Immunol. 187, 1:93-9.

WHO, 2016: Guidelines for the Management of Snakebites. $2^{\text {nd }}$ Ed., WHO Regional Office for South-East-Asia. https://apps.who.int/iris/handle $/ 10665 / 249547$

WHO, 2018: Guidelines for the Production, Control and Regulation of Snake Antivenom Immunoglobulins. Geneva, Switzerland (accessed on 2 July 2019). Available online: https://www. who.int/bloodproducts/snake

Wilson, SC, Vrahas, MS, Berson, L, Paul, E M, 1997: A simple method to measure compartment pressures using an intravenous catheter. Orthopedics 20:403-6.

CDC, 2021: The National Institute for Occupational Safety and Health (NIOSH).

WHO, 2018: Prevalence of snakebite envenoming.https://www.who.int/snakebites/epidemiolog y/en/

Yip, L, 2002: Rational use of crotalidae polyvalent immune Fab (ovine) in the management of crotaline bites. Ann. Emerg. Med. 39:648-52.

Table 1: Site of action of venom, clinical features, tests, and management

\begin{tabular}{|l|l|l|l|}
\hline Site of action & Clinical features & Laboratory tests & Management \\
\hline Local & $\begin{array}{l}\text { Pain } \\
\text { Fang marks } \\
\text { Swelling } \\
\text { Ecchymoses } \\
\text { Lymphadenitis }\end{array}$ & $\begin{array}{l}\text { Antivenom } \\
\text { Compartment syndrome } \\
\text { Rare }\end{array}$ \\
\hline Neurotoxicity & $\begin{array}{l}\text { Ptosis, diplopia } \\
\text { Dysphagia } \\
\text { Bulbar palsy: "drooling" pooling of } \\
\text { pharynx secretions } \\
\text { Dyspnea } \\
\text { Limb weakness }\end{array}$ & $\begin{array}{l}\text { Tensilon test where appro- } \\
\text { priate }\end{array}$ & $\begin{array}{l}\text { Antivenom } \\
\text { Supportive; ventilator support } \\
\text { Anticholinesterase depended on } \\
\text { snake }\end{array}$ \\
\hline Coagulopathy & $\begin{array}{l}\text { Epistaxis, gingival ooze } \\
\text { Bleeding from venipuncture site } \\
\text { Ecchymoses and bruising } \\
\text { Clinically evident bleeding (hemopty- } \\
\text { sis, hematemasis, hematuria, intracra- } \\
\text { nial hemorrhage) }\end{array}$ & $\begin{array}{l}\text { Pecreased fibrinogen } \\
\text { Raised fibrin degradation } \\
\text { proucts, D-dimers }\end{array}$ & $\begin{array}{l}\text { Whole blood clotting test } \\
\text { Thrombocytopenia } \\
\text { Heparin, e-ACA not helpful }\end{array}$ \\
\hline Shock & Hypotension, tachycardia & & $\begin{array}{l}\text { Antivenom, Intravenous fluids } \\
\text { Central pressure monitoring }\end{array}$ \\
\hline
\end{tabular}

